\title{
Inter-integration membrane-reactive distillation for EL synthesis: Equipment development and experimental validating
}

\author{
Wentao Han ${ }^{1}$, Zhenwei Han ${ }^{1}$, Xuechao $\mathrm{Gao}^{2}$, Zhou Hong ${ }^{2}$, Xingang $\mathrm{Li}^{1}$, Hong $\mathrm{Li}^{1}$, \\ Xuehong $\mathrm{Gu}^{2}$, and Xin $\mathrm{Gao}^{1}$ \\ ${ }^{1}$ Tianjin University \\ ${ }^{2}$ Nanjing Tech University
}

March 8, 2021

\begin{abstract}
Ethyl levulinate, one of main derivatives of levulinic acid (LA), is of significant potential as platform chemicals for bio-based materials. The esterification of LA was generally carried out in a conventional batch reactor or in a conventional reactive distillation column. However, traditional methods are hard to deal with equilibrium limited reactions and azeotropic issues. Therefore, the reactive-vapor permeation-distillation (R-VP-D) process, which integrated reaction, distillation and membrane dehydration into one single unit, is proposed in this paper and validated in the pilot-scale experiments. A comparative study is made between a pilot-scale RD column with and without vapor permeation membrane module. Owing to the water-selective membrane and the ingenious design of related apparatuses, the R-VP-D process reveal a superiority in LA conversion of $21.9 \%$ maximum higher than RD without VP process and removing of product water about $53.6 \%$ from VP module, which indicates its promising industrial application in process intensification field.
\end{abstract}

\section{Hosted file}

Manuscript2021_AIChE_MRD_Exp.pdf available at https://authorea.com/users/400106/articles/ 512465-inter-integration-membrane-reactive-distillation-for-el-synthesis-equipmentdevelopment-and-experimental-validating 